\title{
Yasmine Marcil, La fureur des voyages. Les récits de voyage dans la presse périodique (1750-1789)
}

\section{Geza Szasz}

\section{(2) OpenEdition}

1 Journals

\section{Édition électronique}

URL : http://journals.openedition.org/studifrancesi/7926

DOI : 10.4000/studifrancesi.7926

ISSN : 2421-5856

Éditeur

Rosenberg \& Sellier

\section{Édition imprimée}

Date de publication : 1 juillet 2009

Pagination : 397-398

ISSN : 0039-2944

\section{Référence électronique}

Geza Szasz, «Yasmine Marcil, La fureur des voyages. Les récits de voyage dans la presse périodique (1750-1789) », Studi Francesi [En ligne], 158 (LIII | II) | 2009, mis en ligne le 30 novembre 2015, consulté le 08 janvier 2021. URL : http://journals.openedition.org/studifrancesi/7926 ; DOI : https://doi.org/ 10.4000/studifrancesi.7926

Ce document a été généré automatiquement le 8 janvier 2021.

\section{(c) $($ ) $\odot$}

Studi Francesi è distribuita con Licenza Creative Commons Attribuzione - Non commerciale - Non opere derivate 4.0 Internazionale. 


\title{
Yasmine Marcil, La fureur des voyages. Les récits de voyage dans la presse périodique (1750-1789)
}

\author{
Geza Szasz
}

\section{RÉFÉRENCE}

YASMINE MARCIL, La fureur des voyages. Les récits de voyage dans la presse périodique

(1750-1789), Paris, Champion, 2007 («L'Atelier des voyages», 4), pp. 650.

1 La recherche sur la littérature des voyages considère le XVIII siècle comme l'âge d'or des Voyages. La production abondante de cette période constitue l'objet d'un grand nombre d'études portant sur les textes des récits, la représentation des pays et des hommes, l'itinéraire, le rôle du voyage dans «l'élargissement du monde connu». Cependant, une fois le texte ou le voyageur étudié, on passait d'habitude sous silence les différents aspects «sociologiques» de la lecture des récits de voyage, la recherche se limitant dans ce cas surtout à l'analyse des bibliothèques. Une étude profonde des moyens d'accès du public contemporain aux informations sur les nouveaux récits a fait longtemps défaut, alors que la «publicité» littéraire précoce (dont l'abondance peut être comparée à celle des textes), parue dans la presse périodique constitue justement une formidable preuve de l'engouement du public et de la «République des lettres» en général pour les récits de voyage, "genre conquérant», et offre un nouveau terrain d'étude de la représentation des représentations. Cette lacune est comblée par le livre de Yasmine Marcil, consacré à l'analyse des articles de la presse périodique des années '50 et ' $80 \mathrm{du}$ XVIII ${ }^{\mathrm{e}}$ siècle, relatant la parution de récits de voyage. Le sujet ainsi que le corpus analysé paraissent délicats, étant donné par exemple l'opinion des philosophes sur la presse, alors que le public cultivé jugeait déjà indispensable la lecture des journaux. Il s'impose donc pour l'auteur l'évaluation du rôle de médiateur des journalistes littéraires. 
2 Le principal moyen d'analyse consiste à étudier les règles d'écriture des articles («Nouvelles littéraires») dans une vingtaine de périodiques édités d'une part entre 1750 et 1759 et d'autre part entre 1780 et 1789 . La directive du choix a été l'intérêt pour l'annonce et le commentaire des nouveaux livres publiés. Si les journalistes littéraires doivent être considérés sous l'aspect de la médiation, la presse est aussi étudiée comme moyen de communication. Cela nous renseigne sur les contraintes du journaliste au $\mathrm{XVIII}^{\mathrm{e}}$ siècle et, si l'on res-te dans le domaine de la publicité littéraire, des règles du genre du compte rendu. En suivant une logique très claire, on saisit aussi l'ambiguïté du statut du journaliste et des périodiques qui contribuent à la circulation des savoirs et participent en même temps à la construction de l'intérêt des ouvrages. A la base de la recherche se trouvent des études de cas, alors que l'analyse de la critique journalistique s'ordonne autour de deux axes: les règles d'écriture de la recension, et, dans le cas des comptes rendus de récits de voyage scientifique, la question de la validation des faits et des résultats rapportés par le voyageur.

Dans la première partie du volume, l'évocation des questions et problèmes théoriques (terminologie utilisée dans la description d'un récit de voyage, les doutes, très anciens, relatifs au voyage et son récit, le courant critique du xvIII ${ }^{e}$ siècle, les difficultés de la définition du récit de voyage, l'importance, les motifs et les spécificités du récit de voyage scientifique) est suivie par la présentation de l'évolution de la presse française au XVIII ${ }^{e}$ siècle (avec un accent sur les «titres-institutions»). Une étude du lectorat est aussi ébauchée, tout en stipulant que l'on dispose de peu d'informations, mais qu'il devait s'agir d'un public cultivé et, du fait du coût d'abonnement, aisé. En abordant les manières d'écriture sur les récits de voyage, quatre grandes catégories sont distinguées (extrait, compte rendu, nouvelles littéraires, annonce bibliographique - auxquelles s'ajoutent, pour la période des années '80, les nouvelles sur les voyageurs en partance ou déjà partis). Une rapide présentation des évolutions du récit de voyage au XviII ${ }^{\mathrm{e}}$ siècle (le Grand Tour et le voyage pittoresque, l'étude de la montagne, etc.), indispensable pour la mise en contexte, a aussi lieu. Récit de voyage et presse se rencontrent au moment où l'auteur nous conseille prudence dans l'évaluation de leur collaboration: la place du récit de voyage restait tout le temps mineure dans les périodiques - on doit donc relativiser la montée de l'intérêt. Une forte inégalité entre les récits se fait aussi remarquer: certains sont plus, d'autres moins annoncés. (Le palmarès revient, pour les années 1750-1759, au Voyage d'Égypte et de Nubie de Norden, avec 22 annonces, alors qu'au cours des années 1780-1789, le Voyage pittoresque de Sicile de Houël a droit à 48 articles).

4 La deuxième partie («Les règles d'écriture du compte rendu») précise, à propos du travail de critique, que selon la conception des Lumières, la principale mission du journal consistait à instruire le public, même si les idées relatives à la critique n'étaient pas partout pareilles. On rencontre ici un des grands axes du livre: l'étude de trois périodiques emblématiques, L'Année littéraire, le Journal encyclopédique et le Mercure de France (d'abord pour leurs histoires, combats, idées et tons). Fidèlement à l'objectif initial, les «outils» de présentation (citations, paraphrase, suppressions, résumé) sont aussi évoqués. Le compte rendu est désormais, avec ses aspects théoriques (esthétique ou instruction, l'aspect du voyage à saisir) et pratiques (structure, choix et présentation des sujets) au cœur de l'analyse. Tout cela acquiert une importance particulière dès que l'on aborde les motifs de sélection du compte rendu, lui-même nommé travail de synthèse sélectif. D'après l'auteur, ceux-ci sont peu exposés, mais le principal en est sans 
doute le caractère inédit du sujet, alors que les journalistes font preuve de désintérêt à l'égard des chapitres traitant d'histoire naturelle, les traversées océaniques, les détails géographiques, les descriptions des gouvernements, de l'agriculture, du commerce, le déroulement du voyage ou le parcours, bref, à l'égard de tout ce qui rend pour nous le récit de voyage du XVIII ${ }^{\mathrm{e}}$ siècle une source précieuse pour l'histoire des idées.

Quant aux ouvrages sélectionnés et mentionnés ci-dessus, Yasmine Marcil souligne les différences entre les trois grands périodiques (relatives à la rapidité de la publication, le nombre d'articles, l'intérêt et les débats) - et relève un point commun un peu étonnant pour les connaisseurs de l'époque (les années 1780): le désintérêt pour les monuments. (Avec, comme preuve, les commentaires du grand texte de Savary, les Lettres sur l'Égypte, publié en trois volumes en 1785). Il s'ensuit l'étude des règles d'écriture communes et, comme le compte rendu ne peut pas se limiter à une simple présentation, des procédés d'évaluation (positive ou négative).

6 La question de la crédibilité du récit de voyage et du voyageur, qui nous incite à établir des critères de validité et d'authenticité, pousse aussi les journalistes à réfléchir sur les qualités du bon voyageur et du bon récit. La base du jugement sera alors l'organisation du récit et son style (de préférence simple et capable de rendre la lecture agréable), alors que dans le cas des traductions, le style ainsi que les rectifications (des erreurs éventuelles) reviennent au traducteur. Pour cause de crédibilité (et des problèmes de style), l'intérêt des périodiques va surtout aux récits de voyage «récents» (écrits/ publiés au XVIII ${ }^{e}$ siècle), alors que les plus anciens sont en général dépréciés - ce qui ne veut pas dire un enthousiasme égal pour tous les récits de voyage du XvIII ${ }^{e}$ siècle. Cette partie du livre finit par une étude de cas (pour l'évaluation d'un récit de voyage). La présentation du contenu des Lettres sur l'Italie de Dupaty est suivie de celle des réactions dans la presse. Cela nous renseigne à la fois sur le texte, la grille d'analyse de l'auteur et la méthode à suivre si l'on a affaire à un travail de ce type (par exemple dans le cas de la presse d'un pays étranger).

7 La troisième partie du livre est entièrement consacrée aux récits de voyage scientifique, «sous-genre» à succès. Même si la popularité de ce type est sans équivoque, la presse prenant aussi sa part à la diffusion des savoirs, on peut déjà voir sur ce point de nets écarts entre les journaux. Cependant, alors que le discours scientifique est de plus en plus connu et apprécié en grande partie grâce à la presse, les journalistes refusent en général de prendre positions dans les querelles entre savants. Quant à l'étude de la montagne, notamment des Alpes, une des révolutions scientifiques et littéraires du second XVIII ${ }^{e}$ siècle, l'analyse se fait à partir des textes fondamentaux de Saussure et de Dolomieu.

Le livre de Yasmine Marcil nous fournit aussi les preuves de ses constats. Les chercheurs apprécieront sans doute que l'auteur ait inséré dans l'Annexe, entre le texte proprement dit et la Bibliographie extrêmement riche (avec une liste exhaustive et vérifiée des récits de voyage commentés dans la presse périodique, nous renseignant sur les liens entre le Voyage et sa lecture) douze textes parus dans la presse périodique, savamment répartis sur l'ensemble des grands sujets étudiés. Un Index des noms et des titres (de journaux), indispensable dans le cas d'un livre tellement novateur, complète l'ouvrage et contribue à en faire un livre de référence pour longtemps. 